\title{
I 28 Head-to-head Comparison of whole-heart coronary MR and 40/64 slice multidetector-CT angiography for detection of coronary artery stenosis
}

\author{
Anne-Catherine Pouleur, le Polain de Waroux Jean-Benoit, Joelle Kefer, \\ Céline Goffinet, Jean-Louis Vanoverschelde and Bernhard L Gerber*
}

Address: Cliniques Universitaires St. Luc, Brussels, Belgium

* Corresponding author

from I th Annual SCMR Scientific Sessions

Los Angeles, CA, USA. I-3 February 2008

Published: 22 October 2008

Journal of Cardiovascular Magnetic Resonance 2008, I0(Suppl I):A29 doi:10.1 I86/I532-429X-10-SI-A29

This abstract is available from: http://jcmr-online.com/content/I0/SI/A29

(c) 2008 Pouleur et al; licensee BioMed Central Ltd.

\section{Introduction}

Whole-heart magnetic resonance coronary angiography (WH-MRCA) has been recently proposed for non-invasive coronary imaging. Early studies have suggested that WHMRCA might have similarly high diagnostic accuracy for detection of coronary disease as multidetector CT (MDCT). Yet, no direct comparison between both techniques has been performed.

\section{Purpose}

The aim of the present study was therefore to perform a head-to-head comparison of both techniques for detection of significant coronary stenoses using invasive cardiac catheterization as reference standard.

\section{Methods}

Seventy-seven consecutive patients ( $56 \mathrm{M}, 61 \pm 14$ years) prospectively underwent free-breathing 3-dimensional WH-MRCA and 40/64-slice MDCT before cardiac catheterization. WH-MRCA and MDCT images were visually graded by 2 blinded observers and the diagnostic accuracy of both methods for detecting $>50 \%$ luminal diameter stenoses (DS) in segments and vessels $>1.5 \mathrm{~mm}$ size was compared using quantitative angiography (QCA) as reference method.

\section{Results}

MDCT was successfully completed in all 77 patients in < 5 minutes. By contrast, WH-MRCA failed in 9 patients
(12\%) because of poor navigator performance and lasted $20 \pm 4$ minutes $(\mathrm{p}<.01$ vs MDCT). According to QCA, out of 992 segments $>1.5 \mathrm{~mm}$ diameter, 49 presented $>50 \%$ DS. If all segments including non interpretable segments were considered, WH-MRCA had lower sensibility (35/49 or $71 \%$ vs. $45 / 49$ or $92 \%, \mathrm{p}<0.001)$, lower specificity $(644 / 943$ or $68 \%$ vs. $863 / 943$ or $92 \%, p<0.001)$ and accuracy (679/992 or $68 \%$ vs. $908 / 992$ or $92 \%$, p < 0.001 ) for detection of coronary stenosis than MDCT. However if only interpretable segments were considered, the sensitivity (35/37 or $95 \%$ vs. $45 / 46$ or $98 \%, p=0.58)$, specificity (644/689 or $93 \%$ vs. $863 / 917$ or $94 \%, p=0.67)$ and diagnostic accuracy $(679 / 726$ or $94 \%$, vs. $908 / 963$ or $94 \%, p$ $=0.58$ ) of WH-MRCA and MDCT for detection of $>50 \%$ DS was similar. This was also the case on per-vessel basis.

\section{Conclusion}

In the present study CT had higher success rate than $\mathrm{WH}$ MRCA. Therefore on an intention to diagnose basis CT was superior to WH-MRCA. However, the diagnostic accuracy of WH-MRCA on per-segment and per-vessel basis was not statistically different from MDCT if only interpretable segments were considered. 